\title{
Linfadenitis necrotizante histiocitaria. Comunicación de 3 casos
}

\author{
LUIS SEPÚLVEDA P. ${ }^{1}$, FELIPE OLIVARES A. ${ }^{\mathrm{a}}$, \\ ALEKSANDAR MUNJIN P. ${ }^{\mathrm{a}}$, NICOLÁS MORÁN C. ${ }^{\mathrm{a}}$
}

\section{Histiocytic necrotizing lymphadenitis: report of 3 cases}

Histiocytic necrotizing lymphadenitis (also known as Kikuchi-Fujimoto's disease) is an uncommon, benign and self-limiting lymph disease. We report three patients aged 14, 22 and 50 years, who presented with fever and cervical lymphadenopathy, accompanied by skin lesions and joint pain in two of the three cases. One of the patient's sister suffered from histiocytic necrotizing lymphadenitis. Laboratory abnormalities varied and findings included leucopenia, relative neutrophilia, elevated $C$ reactive protein, erythrocyte sedimentation rate and ferritin. Basic laboratory screening tests were performed on all patients to rule out autoimmune and infectious diseases. Lymph node biopsy and subsequent pathological examination were essential to establish the diagnosis. All patients received antibiotics at some point of their hospital stay. Two patients required glucocorticoid treatment, while the remaining case experienced a spontaneous recovery. Its pathogenesis is still unknown, but clinical and histopathological studies suggest a connection with autoimmune diseases. There is no established treatment, but apparently the disease responds to the administration of glucocorticoids.

(Rev Med Chile 2013; 141: 659-663).

Key words: Autoimmunity; Histiocytic Necrotizing Lymphadenitis; KikuchiFujimoto's Disease.
${ }^{1}$ Servicio de Medicina Interna, Hospital Militar de Santiago, Santiago, Chile. anternos de Medicina, Universidad de los Andes, Santiago, Chile.

Recibido el 27 de julio de 2012, aceptado el 14 de diciembre de 2012.

Correspondencia a: Dr. Luis Augusto Sepúlveda Pavez

Avenida Larraín \# 9100, La Reina. Teléfono: +56-99372314 E-mail: lasepulp@gmail. com
L a enfermedad de Kikuchi-Fujimoto (EKF) o linfadenitis necrotizante histiocitaria es una entidad poco frecuente, benigna y autolimitada. Afecta con mayor frecuencia a mujeres jóvenes asiáticas y se caracteriza por presentar fiebre y adenopatías regionales o generalizadas ${ }^{1,2}$.

Las manifestaciones más frecuentes incluyen fiebre prolongada y adenopatías, las cuales habitualmente son cervicales posteriores, sin embargo, pueden aparecer en cualquier localización $\mathrm{n}^{3,4}$.

Reportes de casos asociados a enfermedades como síndrome antifosfolípido, polimiositis, artritis idiopática juvenil, uveítis bilateral, síndrome de Sjögren, vasculitis, hemorragia pulmonar y especialmente lupus eritematoso sistémico (LES), sugieren mecanismos inmunológicos en su patogenia $^{5-12}$. Incluso, un reporte sostiene que la EKF correspondería a una manifestación inicial de LES $^{13}$. De aclararse esta controversia pudiera haber implicancias terapéuticas.

Los pocos casos reportados en nuestro medio $^{14-17}$ motivan este reporte de 3 casos, discutiendo además algunos aspectos diagnósticos y terapéuticos.

\section{Caso 1}

Varón de 14 años, previamente sano, que consultó por adenopatías occipitales, yugulares, submandibulares e inguinales de dos meses de evolución, sensibles, asociadas a fiebre, pérdida de peso, fatiga, anorexia y lesiones máculo-petequiales en el dorso de ambos pies. El hemograma

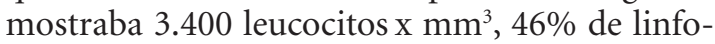


citos y $50 \%$ de neutrófilos. La proteína $\mathrm{C}$ reactiva (PCR) y la velocidad de sedimentación (VHS) fueron de $6,2 \mathrm{mg} / \mathrm{L}$ y $28 \mathrm{~mm} / \mathrm{h}$, respectivamente. El perfil hepático, las pruebas de coagulación y la función renal no mostraron alteraciones. El estudio complementario fue negativo: hemocultivos, urocultivo, coprocultivo, inmunofluorescencia directa para virus respiratorios, serología para virus Epstein-Barr (VEB), Bartonella hensenlae, parvovirus B-19, citomegalovirus (CMV), Toxoplasma gondii y ELISA para VIH. No hubo hallazgos significativos en la tomografía axial computada (TAC) de tórax, abdomen y pelvis, y en la ecocardiografía transtorácica. La ecografía cervical mostró adenopatías bilaterales, tanto en los triángulos cervicales anteriores y posteriores, de aspecto inespecífico.

Durante la hospitalización apareció un exantema máculo-papular en la región malar, tronco y extremidades asociado a artralgias. Los anticuerpos anti-nucleares (ANA) y anti-DNA fueron negativos y los anticuerpos anti-antígenos nucleares extraíbles (ENA) y el factor reumatoide (FR) no presentaron alteraciones. Los niveles de complemento C3 y C4 fueron de $131 \mathrm{mg} / \mathrm{dL}$ y $45 \mathrm{mg} / \mathrm{dL}$ respectivamente. Se realizó biopsia de adenopatía cervical que reveló distorsión de la arquitectura, focos de necrosis fibrinoide y restos apoptóticos rodeados de abundantes histiocitos, compatible con linfadenitis necrotizante histiocitaria. El cuadro se resolvió espontáneamente en 1 mes y tras un año de seguimiento no ha presentado recurrencias.

\section{Caso 2}

Mujer de 22 años, sana. Consultó por aumento de volumen cérvico-parotídeo derecho sensible, de 3 meses de evolución y refractario a antiinflamatorios. Fue interpretado como síndrome de mononucleosis infecciosa y por su persistencia se le indicó empíricamente amoxicilina-ácido clavulánico sin mejoría clínica. Finalmente, le indicaron glucocorticoides con resolución completa del cuadro.

Dos meses después consultó nuevamente por fiebre asociado a múltiples adenopatías submandibulares sensibles e induradas, con aumento de volumen parotídeo ipsilateral. Presentaba PCR de $41 \mathrm{mg} / \mathrm{L}$ y hemograma con neutrofilia relativa. Las pruebas hepáticas y LDH estaban en rangos normales.
Se solicitó TAC cervical que mostró aumento de volumen parotídeo y de grupos ganglionares cervicales bilaterales, ambos de predominio derecho. Además se informó un probable flegmón cervical. Se reiniciaron los antibióticos (ceftriaxona más clindamicina) sin respuesta. Dada la persistencia del cuadro, se escaló a esquema antibiótico de segunda línea (vancomicina más levofloxacino) y se amplió estudio con TAC de tórax-abdomen-pelvis que informó linfonodos precarinales de $6 \mathrm{~mm}$, sin otros hallazgos. El estudio inmunológico con ANA e infeccioso para VEB, CMV, VIH, sífilis, Toxoplasma gondii y Bartonella hensenlae fueron negativos. Finalmente, se realizó biopsia de ganglio cervical que reveló hallazgos compatibles con linfadenitis necrotizante histiocitaria. Se suspendieron los antibióticos y se indicó tratamiento con prednisona $30 \mathrm{mg}$ al día con disminución progresiva de las dosis a partir de la segunda semana, completando 1 mes de tratamiento. La fiebre cedió a las $24 \mathrm{~h}$ tras el inicio de la terapia y no ha presentado recurrencias a los 6 meses de seguimiento.

\section{Caso 3}

Mujer de 50 años con historia de poliartralgias y tabaquismo crónico activo. Refiere cuadro de 2 meses caracterizado por fiebre persistente, sudoración nocturna, baja de peso, poliartralgias en extremidades inferiores y aumento de volumen cervical derecho sensible. Relata antecedente de hermana con EKF y contacto directo con gato. Consultó en Servicio de Urgencias, donde le realizaron ecografía cervical que mostraba múltiples linfonodos cervicales a derecha. Le indicaron amoxicilina-ácido clavulánico durante 5 días sin mejoría clínica por lo que se hospitalizó. En el examen físico destacaba una adenopatía cervical derecha de $5 \times 6 \mathrm{~cm}$, sensible y sin visceromegalia. El hemograma mostró VHS de $56 \mathrm{~mm} / \mathrm{h}$ y la PCR y ferritina fueron de $77 \mathrm{mg} / \mathrm{L}$ y $1.789 \mathrm{ng} / \mathrm{mL}$ respectivamente. El TAC de cuello, tórax, abdomen y pelvis, además de constatar adenopatías cervicales y mediastínicas, reveló opacidades pulmonares en vidrio esmerilado. Paralelamente, se descartó infección por VIH, VEB, CMV, virus hepatitis $\mathrm{B}$ y C, sífilis y Toxoplasma gondii. La IgM para Bartonella hensenlae fue negativa, mientras que la IgG resultó positiva (1/64). Se indicó azitromicina $1 \mathrm{~g}$ al día 
durante 6 días, sin respuesta. El estudio inmunológico fue prácticamente normal, solamente con un ANA positivo a título bajo (1/40). Se decidió realizar biopsia de la adenopatía cervical. Los estudios para hongos y bacterias resultaron negativos, mientras que el estudio histológico reveló linfadenitis necrotizante histiocitaria. Se inició prednisona $30 \mathrm{mg}$ al día durante tres semanas con disminución progresiva de las dosis completando 45 días de terapia. Los síntomas desaparecen a las $48 \mathrm{~h}$ de tratamiento. Dos meses después asiste a control con TAC de tórax que informó aumento de imágenes en vidrio esmerilado. Se realizó biopsia pulmonar revelando hallazgos compatibles con neumonía intersticial usual en fase tardía. A los 6 meses de seguimiento no ha presentado sintomatología respiratoria ni alteraciones significativas en las pruebas de función pulmonar.

\section{Discusión}

En los casos expuestos el motivo de consulta principal fue fiebre prolongada y adenopatías cervicales, lo que es consistente con lo previamente reportado ${ }^{3,4}$. Las adenopatías pueden aparecer en cualquier localización, incluso a nivel intraparotídeo $^{18}$, lo que podría explicar el aumento de volumen en esa región presentado por la mujer del caso 2.

En el primer caso se describe exantema malar, artralgias y petequias en los pies. Estas manifestaciones son comunes en algunas enfermedades autoinmunes. El compromiso cutáneo es variable y está presente en un tercio de los casos. Se han descrito exantemas maculares (especialmente malar), papulares, nodulares, eritema polimorfo, erupciones pustulares, úlceras orales, descamación y erosiones ${ }^{19-23}$. Estas manifestaciones hacen plantear otras enfermedades del tejido conectivo en el diagnóstico diferencial.

El LES comparte con la EKF características clínicas, epidemiológicas e histológicas y ha sido observado tanto en forma previa, concomitante o posterior a su aparición ${ }^{24-27}$. La existencia de una asociación entre ambas entidades, o si constituye una etapa de la misma enfermedad, aún es motivo de debate. Algunos investigadores plantean que la EKF podría corresponder simplemente a una linfadenitis lúpica como una forma de presentación inicial de LES localizado ${ }^{13,28,29}$. Para clarificar esta situación, $\mathrm{Hu}$ y cols, analizaron clínica e histológicamente a 18 mujeres con EKF y LES, no encontrando relación entre ambas entidades y concluyen que los pacientes estudiados como EKF correspondieron más bien a linfadenitis lúpica que cumplían con algunos de los criterios histológicos de EKF, sin embargo, seis de ellas tenían indiscutiblemente EKF concomitante al LES $^{30}$. Ello muestra que esta asociación aún es controversial y de aclararse podría eventualmente tener implicancias terapéuticas.

Se ha planteado una relación entre la patogenia de la EKF con mecanismos autoinmunes probablemente desencadenados por agentes infecciosos, que presentan mimetismo molecular con antígenos propios ${ }^{31}$. A pesar que se han reportado casos de EKF concomitantes a VEB, virus de linfoma T humano 1 (HTLV-1), virus herpes $6 \mathrm{y}$ 8, parvovirus B19, CMV, parainfluenza y Yersinia enterocolítica, actualmente el rol de estos agentes es discutido, a tal punto que podrían corresponder meramente a un hallazgo serológico o a un falso positivo $^{32-37}$. Otra hipótesis sugiere como patogenia una respuesta inmune desproporcionada contra autoantígenos expresados en células apoptóticas de pacientes genéticamente susceptibles ${ }^{38}$.

Una agregación familiar podría explicar la hermana con EKF en el tercer caso y al mismo tiempo sustentar la hipótesis anteriormente mencionada, sin embargo, existen escasos casos reportados al respecto ${ }^{39-42}$ y solamente en dos estudios los pacientes compartían genotipos del sistema mayor de histocompatibilidad humana ${ }^{39,40}$, desconociéndose si cumplen algún rol.

En el segundo y tercer caso los pacientes respondieron al uso de glucocorticoides, mientras que en el primero se resolvió espontáneamente. La EKF es, generalmente, de curso autolimitado y ningún tratamiento efectivo ha sido establecido. En cuadros severos o persistentes, los glucocorticoides orales han mostrado mejoría al acortar la duración de los síntomas ${ }^{43-46}$, sin existir estudios randomizados al respecto. Un estudio trató con bolos de metilprednisolona a 13 pacientes, observando resolución de la fiebre en $24 \mathrm{~h}^{46}$. También existen algunos reportes que muestran una rápida respuesta al tratamiento con hidroxicloroquina ${ }^{47,48}$. La alta tasa de resolución espontánea conduce, por el momento, a reservar el tratamiento para cuadros prolongados o severos.

La recurrencia de la EKF ha sido reportada 
con frecuencias que varían entre $4 \%$ y $20 \%$. Probablemente esta disparidad podría ser explicada por una subestimación, motivada por pérdida de seguimiento posterior de pacientes. En otro reporte se ha relacionado la presencia de títulos elevados de ANA, como un factor que predice recurrencia ${ }^{49}$.

Lo inespecífico en su presentación, la patogenia poco esclarecida, su asociación controversial con enfermedades autoinmunes y la falta de un tratamiento validado, determinan que la EKF siga siendo un desafío para el médico tratante.

\section{Referencias}

1. Kikuchi M. Lymphadenitis showing focal reticulum cell hyperplasia with nuclear debris and phagocytosis. Nippon Ketsueki Gakkai Zasshi 1972; 35: 379-80.

2. Fujimoto Y, Kosima Y, Yamaguchi K. Cervical subacute necrotizing lymphadenitis. A new clinicopathologic agent. Naika 1972; 20: 920-7.

3. Dorfman RF, Berry GJ. Kikuchi's histiocytic necrotizing lymphadenitis: an analysis of 108 cases with emphasis on differential diagnosis. Semin Diagn Pathol 1988; 5: 329-45.

4. Kucukardali Y, Solmazgul E, Kunter E, Oncul O, Yildirim S, Kaplan M. Kikuchi-Fujimoto Disease: analysis of 244 cases. Clin Rheumatol 2007; 26: 50-4.

5. Pappaioannou G, Speletas M, Kaloutsi V, PavlitouPsiontsi A. Histiocytic necrotizing lymphadenitis (Kikuchi-Fujimoto disease) associated with antiphospholipid syndrome: case report and literature review. Ann Hematol. 2002; 81: 732-5.

6. Wilkinson CE, Nichol F. Kikuchi-Fujimoto disease associated with polymyositis. Rheumatology (Oxford) 2000; 39: 1302-4.

7. Ramanan A, Wynn R, Kelsey A, Baildam E. Systemic juvenile idiopathic arthritis, Kikuchi's disease and haemophagocytic lymphohistiocytosis: is there a link? case report and review of the literature. Rheumatology (Oxford). 2003; 42: 596-8.

8. Taguri AH, McIlwaine GG. Bilateral panuveitis: a possible association with Kikuchi-Fujimoto disease. Am J Ophthalmol 2001; 132: 419-21.

9. Soy M, Peynirci H, Bilgi S, Adali MK, Güresci S. KikuchiFujimoto disease coexisted with Sjogren's syndrome. Clin Rheumatol 2007; 26 (4): 607-8.

10. Belagavi CS, Kubeyinje EP. Recurrent cutaneous necrotizing vasculitis in a patient with Kikuchi's disease: case report. East Afr Med J 1997; 74: 747-8.

11. Wong CY, Law GT, Shum TT, Wong KY, Li YK. Pulmonary haemorrhage in a patient with Kikuchi disease.
Monaldi Arch Chest Dis 2001; 56: 118-20.

12. Martínez-Vázquez C, Hughes G, Bordon J, Alonso-Alonso J, Anibarro-García A, Redondo-Martínez E, et al. Histiocytic necrotizing lymphadenitis, Kikuchi-Fujimoto's disease, associated with systemic lupus erythemotosus. Q J Med 1997; 90: 531-3.

13. Bosh X, Guilabert A, Miquel R, Campo E. Enigmatic Kikuchi-Fujimoto disease: a comprehensive review. Am J Clin Pathol 2004; 122: 141-52.

14. Etcheverry R, Armas R, Martínez V. Linfoadenitis necrotizante subaguda (enfermedad de Kikuchi y Fujimoto). Rev Med Chile 1990; 118: 431-6.

15. Rohmann I, Bentjerodt R. Linfoadenitis necrotizante de Kikuchi: Histopatología en 2 casos. Rev Med Chile 1988; 116: 929-33.

16. Tordecilla J, Medina M, Ávila R, Campbell M. Enfermedad de Kikuchi-Fujimoto. Rev Chil Pediatr 2002; 73 (5): 483-8. Disponible en: www.scielo.cl/scielo. php? script $=$ sci_arttext\&pid $=S 0370-41062002000$ 500006\&lng=es.doi: $10.4067 /$ S0370-41062002000500006 [Consultado el 21 de abril de 2012].

17. Gac P, Cabané P, Franco C, Amat J, Rossi R, Huidobro F, et al. Tumor cervical por enfermedad de Kikuchi. Una entidad clínica a la que no estamos habituados. Caso clínico. Rev Med Chile 2008; 136: 209-16.

18. Kuo T, Jung SM, Wu WJ. Kikuchi disease of intraparotid lymph nodes presenting as a parotid gland tumor with extranodal involvement of salivary gland. Histopathology 1996; 28: 185-8.

19. Yasukawa K, Matsumura T, Sato-Matsumura KC, Yakahashi T, Fujioka Y, Kobayashi H, et al. Kikuchi's disease and the skin: case report and review of the literature. $\mathrm{Br}$ J Dermatol 2001; 144: 885-9.

20. Kaur S, Thami GP, Mohan H, Kanwar A. Kikuchi disease with facial rash and erythema multiforme. Pediatr Dermatol 2001; 18: 403-5.

21. Spies J, Foucar K, Thompson C, LeBoit P. The histopathology of cutaneous lesions of Kikuchi's disease: a report of 5 cases. Am J Surg Pathol 1999; 23: 1040-7.

22. Kim KJ, Jee MS, Chang SE, Choi JH, Sung KJ, Moon KC, et al. Kikuchi-Fujimoto disease with papulopustular skin manifestations. Clin Exp Dermatol 2003; 28: 142-4.

23. Imai K, Yokozeki H, Nishioka K. Kikuchi's disease (histiocytic necrotizing lymphadenitis) with cutaneous involvement. J Dermatol 2002; 29: 587-92.

24. Mahajan T, Merriman R, Stone M. Kikuchi-Fujimoto disease (histiocytic necrotizing lymphadenitis): report of a case with other autoimmune manifestations. Proc (Bayl Univ Med Cent) 2007; 20: 149-51.

25. López C, Oliver M, Olavarria R, Sarabia MA, Chopite M. Kikuchi-Fujimoto necrotizing lymphadenitis associated 
with cutaneous lupus erythematosus: a case report. Am J Dermatopathol 2000; 22 (4): 328-33.

26. Chen HC, Lai JH, Huang GS, Gao HW, Chen CH, Kuo SY, et al. Systemic lupus erythematosus with simultaneous onset of Kikuchi-Fujimoto's disease complicated with antiphospholipid antibody syndrome: a case report and review of the literature. Rheumatol Int 2005; 25 (4): 303-6.

27. Santana A, Lessa B, Galrao L, Lima I, Santiago M. Kikuchi-Fujimoto's disease associated with systemic lupus erythematosus: case report and review of the literature. Clin Rheumatol 2005; 24 (1): 60-3.

28. Imamura $\mathrm{M}$, Ueno $\mathrm{H}$, Matsuura A. An ultrastructural study of subacute necrotizing lymphadenitis. Am J Pathol1982; 107: 292-9.

29. Jiménez JM, Llorente EM, Fuentes F, de Miguel F, Álvarez R. Enfermedad de Kikuchi-Fujimoto y su asociación a lupus eritematoso sistémico. An Med Interna (Madrid) 2001; 18: 429-31.

30. Hu S, Kuo TT, Hong HS. Lupus lymphadenitis simulating Kikuchi's lymphadenitis in patients with systemic lupus erythematosus: a clinicopathological analysis of six cases and review of the literature. Pathol Int 2003; 53: 221-6.

31. Marrack P, Kappler J, Kotzin BL. Autoimmune disease: why and where it occurs. Nat Med 2001; 7 (8): 899-905.

32. Chiu CF, Chow KC, Lin TY, Tsai MH, Shih CM, Chen LM. Virus infection in patients with histiocytic necrotizing lymphadenitis in Taiwan: detection of EpsteinBarr virus, type I human T-cell lymphotropic virus, and parvovirus B19. Am J Clin Pathol 2000; 113: 774-81.

33. Sumiyoshi Y, Kikuchi M, Ohshima K, Yoneda S, Kobari S, Eizuru Y, et al. Human herpesvirus-6 genomes in histiocytic necrotizing lymphadenitis (Kikuchi's disease) and other forms of lymphadenitis. Am J Clin Pathol 1993; 99: 609-14.

34. Georges TI, Jones CD, Zehnder JL, Warnke RA, Dorfman RF. Lack of human herpesvirus 8 and Epstein-Barr virus in Kikuchi's histiocytic necrotizing lymphadenitis. Hum Pathol 2003; 34: 130-5.

35. Hollingsworth HC, Peiper SC, Weiss LM, Raffeld M, Jaffe ES. An investigation of the viral pathogenesis of Kikuchi-Fujimoto disease: lack of evidence for EpsteinBarr virus or human herpesvirus type 6 as the causative agents. Arch Pathol Lab Med 1994; 118: 134-40.

36. Huh J, Chi HS, Kim SS. A study of the viral etiology of histiocytic necrotizing lymphadenitis (Kikuchi-Fujimoto disease). J Korean Med Sci 1998; 13: 27-30.

37. Heikens J, Tel W, van de Stadt J. Kikuchi's lymphadenitis: report of a Yersinia enterocolitica-associated case and an overview of a etiology and clinical outcome. Neth JMed 1992; 41: 222-8.

38. Botto M. Links between complement deficiency and apoptosis. Arthritis Res 2001; 3 (4): 207-10.

39. Stasiuk A, Teschke S, Williams GJ, Seftel MD. KikuchiFujimoto disease: lymphadenopathy in siblings. CMAJ 2001; 183: E58-60.

40. Amir AR, Amr SS, Sheikh SS. Kikuchi-Fujimoto Disease: report of familial occurrence in two human-leukocyte antigen identical non-twin sisters. J Intern Med 2002; 252: 79-83.

41. Ifeacho S, Aung T, Akinsola M. Kikuchi-Fujimoto disease: a case report and review of the literature. Cases J 2008; 1: 187.

42. Atarashi K, Yoshimura N, Nodera H, Tsukimoto K, Beppu H, Kanayama M. Recurrent histiocytic necrotizing lymphadenitis (Kikuchi's disease) in an human $\mathrm{T}$ lymphotropic virus type I carrier. Intern Med 1996; 35 : 821-5.

43. Yalcin S, Toprak SK, Erismis B, Altundag O, Ozdemir H, Topcuoglu N. Management of Kikuchi-Fujimoto disease using glucocorticoid: a case report. Case Report Med 2011; 2011: 230840. Epub 2011 Aug 14.

44. Jang YJ, Park KH, Seok HJ. Management of Kikuchi's disease using glucocorticoid. J Laryngol Otol 2000; 114: 709-11.

45. Lee KY, Yeon YH, Lee BC. Kikuchi-Fujimoto disease with prolonged fever in children. Pediatrics 2004; 114: 752-6.

46. Yoshioka K, Miyashita T, Nakamura T, Inoue T, Yamagami K. Treatment of histiocytic necrotizing lymphadenitis (Kikuchi's disease) with prolonged fever by a single course of methylprednisolone pulse therapy without maintenance therapy: experience with 13 cases. Intern Med 2010; 49: 2267-70.

47. Rezai K, Kuchipudi S, Chundi V, Ariga R, Loew J, Sha BE. Kikuchi-Fujimoto disease: hydroxychloroquine as a treatment. Clin Infect Dis 2004; 39: 124-6.

48. Chen PH, Huang YF, Tang CW, Wann SR, Chang HT. Kikuchi-Fujimoto disease: an amazing response to hydroxychloroquine. Eur J Pediatr 2010; 169: 1557-9.

49. Song JY, Lee J, Park DW, Sohn JW, Suh SI, Kim IS, et al. Clinical outcome and predictive factors of recurrence among patients with Kikuchi's disease. Int J Infect Dis 2009; 13: 322-6. 